\title{
El enfoque terapéutico del cáncer renal: una nueva oportunidad de coordinación entre la Urología Oncológica y la Oncología médica
}

\author{
Gelabert Mas A. \\ Servicio y Cátedra de Urología. Hospital del Mar. UAB Barcelona. \\ Coordinador Nacional del Grupo de Urología Oncológica AEU.
}

Actas Urol Esp. 2007;31(8):805-806

$\mathrm{E}^{1}$ carcinoma de células renales representa alrededor del $95 \%$ de todos los tumores del riñón. Su incidencia sigue incrementándose, en especial en áreas industrializadas, sin que se conozcan de manera clara los motivos reales, aunque parece seguro que intervienen tanto la mejora en técnicas diagnósticas como razones ambientales, entre otras, el tabaco y la dieta.

Las técnicas de exploración por imagen ambulatorias no agresivas, ecografia abdominal específicamente, son suficientes para el diagnóstico y estadificación, aunque no garantizan un diagnóstico precoz, ya que alrededor de una cuarta parte de los pacientes tendrá enfermedad avanzada en el momento del diagnóstico.

La principal opción terapéutica para los pacientes que padecen esta enfermedad se basa en la cirugía cuando el tumor está localizado en el riñón, asegurando esta técnica un porcentaje elevado de curaciones. De manera distinta ocurre cuando la enfermedad se extiende más allá del riñón, siendo escasas las posibilidades de alcanzar supervivencias de larga duración, en estos casos la mediana de supervivencia raramente supera los 12 meses, debido a la resistencia que presenta este tumor a los tratamientos oncológicos sistémicos convencionales. El cáncer renal es un tumor clásicamente quimiorresistente; hasta el momento ninguna terapia adyuvante ha demostrado reducir las posibilidades de recaída; la radioterapia tras la cirugia tampoco aporta un beneficio claro, ni siquiera en aquellos pacientes con enfermedad ganglionar o resección incompleta.

La cirugía radical supone la única terapia eficaz potencialmente curativa del cáncer de riñón localizado. También está indicada en estadios regionalmente más avanzados; por ejemplo, en los tumores que invaden la vena cava o que presentan mínima enfermedad adenopática. En los tumores de menos de $4 \mathrm{~cm}$ de diámetro y situados en la periferia del parénquima, que no afectan al hilio renal, la nefrectomía parcial reviste las características de radicalidad y debe aplicarse de manera reglada y sistemática; en cambio en los pacientes en donde la nefrectomía radical supone dejarlos con una función renal deficitaria y precisar de diálisis, como por ejemplo en riñones únicos o tumores bilaterales, la nefrectomía parcial debe ser electiva si ello es posible, y si no es posible debe exponerse la situación al paciente para que él asuma los riegos de la misma.

En la década de los 80, cuando empezaron a sedimentarse los principios de la cirugía oncológica, existía una opinión bastante generalizada que la nefrectomía jugaba un papel limitado en la historia natural del cáncer renal, debido a que la enfermedad a distancia era finalmente la responsable de la muerte; pero con el paso de los años, en estos últimos tres lustros, hemos asistido a un renovado interés por la cirugía del tumor primario en la enfermedad metastásica, en un intento de reducir la sintomatología local del tumor primario, controlar la producción ectópica de hormonas-Iike e incluso de lograr un aumento modesto, pero significativo, de la supervivencia. Algunos pacientes con enfermedad monometastásica incluso en sistema nervioso central, pueden lograr una supervivencia prolongada mediante nefrectomía y resección quirúrgica de las metástasis; existe evidencia de que los pacientes que presentan un intervalo amplio entre la nefrectomía inicial y la progresión metastásica son los que más se benefician de esta maniobra.

Los últimos acontecimientos terapéuticos sobre el cáncer renal han aportado muchos cambios en los paradigmas del enfoque terapéutico y han servido como lanzadera para la aprobación de tratamientos nuevos, aún siendo un tumor relativamente poco frecuente. 
Es conocida la constatación de regresiones espontaneas en un pequeño número de pacientes y también la aparición de progresiones, muchos años después de la realización de nefrectomías en pacientes con tumores "teóricamente" localizados, ello ha revelado el papel muy importante del sistema inmunológico en la evolución de estos tumores. El tratamiento con moduladores del sistema inmunológico, como interleukina-2 a altas dosis como estrategia más utilizada en EEUU y con interferón como el tratamiento de elección en Europa, está suficientemente apoyado por datos clínico-patológicos y por esto mismo se ha aprobado su uso por las principales agencias reguladoras de fármacos. Existen estudios bien contrastados en la literatura médica mundial que han demostrado la existencia de respuestas completas de muy larga duración en pacientes con cáncer renal metastásico tratados con interleukina-2 intravenosa, que dada su muy dilatada duración las etiquetamos de curaciones; si bien su uso no ha sido generalizado de manera intensa, ni implementada por los dispositivos asistenciales de manera sistemática; podemos decir que su uso clínico queda circunscrito a grupos muy definidos que tradicionalmente han sido y son muy activos en la aplicación de esta terapia inmunitaria.

Pero volviendo al aspecto del papel de la Cirugía, la nefrectomía en pacientes con carcinoma renal metastásico con fines, no exclusivamente diagnósticos, sino terapéuticos, ha trastocado uno de los paradigmas de la oncología médica, que daba por asumido que no debe extirparse el tumor primario en los tumores avanzados/diseminados; existe la excepción de otro tumor urológico: los tumores testiculares, pero en estos la orquiectomía tiene plena justificación para el diagnóstico y además sabemos de la mala distribución intersticial de la quimioterapia en el teste. Existen, dos estudios aleatorios realizados en pacientes con cáncer renal metastásico que demuestran que la cirugía (nefrectomía) aumenta la supervivencia de estos pacientes si se parte de un buen performance status, indistintamente de su interés diagnóstico o paliativo.

Los profundos conocimientos de la citogenética de los distintos tipos de cáncer renal, y de manera especial en el de células claras, ha posibilitado que se usara este tumor como el objetivo más adecuado para probar las distintas dianas terapéutica nuevas, aún no siendo un tumor excesivamente frecuente. En estos momentos hay más de 30 nuevos fármacos ensayándose como tratamiento del cáncer renal, esta cifra es con toda seguridad demasiado superior a lo que cabría esperar de acuerdo a su frecuencia pero la explicación que asumimos es por la gran cantidad de dianas moleculares bien conocidas y caracterizadas en este tumor y que hasta hace poco había una ausencia casi total de opciones terapéuticas después de la cirugía.

Por su actualidad podemos analizar la angiogénesis. Desde el año 1971 Folkman señalaba el papel de la angiogénesis como un aspecto crítico para la progresión de la historia de los tumores sólidos; han tenido que pasar cerca de tres décadas para demostrar la eficacia clínico-asistencial de los inhibidores de la angiogénesis (bevacizumab) en pacientes con cáncer renal, adelantándose de manera clara, y en cierta manera sorpresiva, a su experimentación con otros tumores sólidos más frecuentes.

En estos últimos años ya se han aprobado la utilización de otros fármacos tanto por parte de la FDA como la EMEA que actúan bloqueando las vías de señalización de VEGF y Ras, (sunitinib) y (sorafenib).

Con todas estas realidades asistenciales nos situamos ante un nuevo escenario en la actividad clínica diaria: la cirugía en la cáncer renal, que siempre ha tenido un papel preponderante, lo sigue teniendo con mayor intensidad si cabe y además en las situaciones clínicas de tumores avanzados actualmente la podemos justificar con datos de la evidencia científica ya que en estos casos la terapia médica adyuvante consigue mejores resultados cuando menor es la carga tumoral existente, es decir, y traduciendo estos términos a la gestión asistencial integral del paciente neoplásico: la planificación coordinada de los momentos terapéuticos entre la Urología Oncológica y la Oncología Médica debe permitir la excelencia asistencial a un nuevo grupo de pacientes Uro-Oncológicos, los afectos de Cáncer renal, que hasta hace muy poco carecian de esperanzas más allá de la cirugía si esta no podía ser curativa.

Correspondencia autor: Dr. A. Gelabert Mas

Servicio y Cátedra de Urología. Hospital del Mar

Passeig Marítim, 25-29 - 08003 Barcelona. Tel.: 932483000

E-mail autor: agelabert@imas.imim.es

Información artículo: Editorial 